Service social

\title{
Le travail des baluchonneuses accompagnant une personne atteinte de démence de type Alzheimer : une responsabilité morale?
}

\author{
Sophie Éthier, Catherine Gagnon-Grégoire et Janine Dupont
}

Volume 60, numéro 2, 2014

URI : https://id.erudit.org/iderudit/1027991ar

DOI : https://doi.org/10.7202/1027991ar

Aller au sommaire du numéro

Éditeur(s)

École de service social de l’Université Laval

ISSN

1708-1734 (numérique)

Découvrir la revue

Citer cet article

Éthier, S., Gagnon-Grégoire, C. \& Dupont, J. (2014). Le travail des

baluchonneuses accompagnant une personne atteinte de démence de type

Alzheimer : une responsabilité morale ? Service social, 60(2), 71-85.

https://doi.org/10.7202/1027991ar
Résumé de l'article

Les baluchonneuses partagent le quotidien de personnes atteintes de démence de type Alzheimer pour une durée de 4 à 14 jours en l'absence de l'aidant familial. Cet article présente une étude visant à mieux comprendre leur travail dans la perspective d'un modèle théorique sur la responsabilité morale. Les données ont été recueillies auprès de 20 baluchonneuses à l'aide d'entrevues face à face, téléphoniques et de groupe. Les résultats font ressortir qu'à travers les fondements derrière leur choix de devenir baluchonneuses - l'actualisation de leur responsabilité, l'évolution de la réponse à l'appel de la responsabilité, la singularisation de la responsabilité, la transformation de leur relation avec l'aidé, la variation de la relation entretenue avec les aidants, leur implication dans la relation aidant-aidé et la gestion de la médication et des soins médicaux -, les baluchonneuses assument une responsabilité morale en partie différente, mais néanmoins très semblable, à celle des aidants familiaux. 


\title{
Le travail des baluchonneuses accompagnant une personne atteinte de démence de type Alzheimer : une responsabilité morale?
}

\author{
ÉTHIER, Sophie \\ GAGNON-GRÉGOIRE, Catherine \\ DUPONT, Janine
}

Cette étude a été rendue possible grâce au soutien financier du Fonds de démarrage des nouveaux professeurs de l'Université Laval.

\section{RÉSUMÉ}

Les baluchonneuses partagent le quotidien de personnes atteintes de démence de type Alzheimer pour une durée de 4 à 14 jours en l'absence de l'aidant familial. Cet article présente une étude visant à mieux comprendre leur travail dans la perspective d'un modèle théorique sur la responsabilité morale. Les données ont été recueillies auprès de 20 baluchonneuses à l'aide d'entrevues face à face, téléphoniques et de groupe. Les résultats font ressortir qu'à travers les fondements derrière leur choix de devenir baluchonneuses-l'actualisation de leur responsabilité, l'évolution de la réponse à l'appel de la responsabilité, la singularisation de la responsabilité, la transformation de leur relation avec l'aidé, la variation de la relation entretenue avec les aidants, leur implication dans la relation aidant-aidé et la gestion de la médication et des soins médicaux -, les baluchonneuses assument une responsabilité morale en partie différente, mais néanmoins très semblable, à celle des aidants familiaux.

Mots-clés: démence de type Alzheimer, Baluchon Alzheimer, responsabilité morale, répit, baluchonneuses.

\begin{abstract}
The baluchonneuses (homecarers) provide services consisting in sharing the daily lives of people with dementia-type Alzheimer's, for periods of 4 to 14 days, to replace their family caregivers. The paper presents results from a study aimed at understanding their work by building on a "moral responsibility" theoretical perspective. Data was gathered from 20 baluchonneuses through phone and face-to-face interviews, as well as a focus-group. Results show that in many respects-namely, their choice to become a baluchonneuse; the implementing of their responsibilities; their response to the call for responsibility; the singularizing of responsibility; their changing relationship to the cared-for person; their evolving relationship to the caregiver; their involvement in the caregiver-care receiver relationship; and the managing of medication and healthcare-the baluchonneuses assume a moral responsibility that differs, yet is also very similar to that of a family caregiver.
\end{abstract}

Key words: Alzheimer's disease, Baluchon Alzheimer, moral responsibility, respite, baluchonneuses. 


\section{INTRODUCTION}

Les besoins des aidants d'une personne atteinte de démence de type Alzheimer (DTA) sont de trois ordres: besoin de soutien, besoin de répit et besoin d'informations et de compétences (Caisse nationale de solidarité pour l'autonomie (CNSA), 2008). Le besoin de répit est clairement reconnu dans la première Politique de soutien à domicile québécoise Chez soi : Le premier choix (ministère de la Santé et des Services sociaux (MSSS), 2003) et réitéré dans la première politique québécoise du vieillissement, Vieillir et vivre ensemble (MSSS, 2012). Quatre services de répit sont abordés ici. Le premier, le centre de jour, offre des activités hors domicile « dans un milieu protégé pour des personnes âgées qui présentent une variété de types d'incapacités » (Savard, 2008, p. 5). Le second service de répit est l'hébergement temporaire qui consiste « à offrir [...] un milieu de vie substitut ainsi que les soins et services requis par leur état pour une période prédéterminée » (Agence de la santé et des services sociaux de la Capitale-Nationale (ASSSCN), 2011, p. 5). Le troisième, le service de présence-surveillance ${ }^{1}$ offert à domicile, est défini par «les activités normales de garde lorsqu'un proche qui habite avec une personne ayant une incapacité doit s'absenter occasionnellement de son domicile pour diverses activités » (MSSS, 2003, p. 42). Le service de répit offert par Baluchon Alzheimer (BA) consiste à des périodes d'accompagnement (baluchonnages) de 24 heures sur 24 pendant 4 à 14 jours consécutifs. Ainsi, contrairement au centre de jour et à l'hébergement temporaire qui impliquent un déplacement pouvant engendrer une désorientation ou de la nervosité chez la personne atteinte de DTA (Bégin et al., 2004 ; Racine, 2012), le modèle de BA lui permet de rester à domicile et de maintenir son rythme de vie habituel (Lucet, 2011 ; ministère des Solidarités et de la Cohésion sociale (MSCS), 2011). Durant leur séjour, les baluchonneuses s'occupent de l'ensemble des soins requis par l'aidé, incluant, s'il y a lieu, les visites médicales et la gestion de la médication en plus d'accomplir des tâches ménagères. Aussi, elles tiennent un journal d'accompagnement contenant leurs observations et recommandations qui est remis à l'aidant au terme du baluchonnage (Lucet, 2011 ; MSCS, 2011), ce que le service de présencesurveillance n'offre pas. II est reconnu que BA répond mieux aux besoins spécifiques des familles (Ducharme et al., 2013; MSCS, 2011) qui souhaitent que leur proche demeure à la maison lors du répit et préfèrent un répit de plusieurs jours consécutifs spécialement lors de la période des vacances (Binhas, 2010).

Mais que sait-on de l'expérience particulière de ceux qui répondent aux besoins de répit des aidants familiaux ? Peu de données sont disponibles à cet égard. Le manque de temps, de formation, de ressources financières et humaines ainsi que les conflits relationnels avec la famille de l'aidé constituent les principales difficultés nommées par ces intervenants (Damasse et al., 2003 ; Éthier et Corbeil, 2003 ; Mackenzie et Peragine, 2003 ; Armstrong-Stassen et Cameron, 2005 ; Pitrou et al., 2006 ; Jansen et al., 2009 ; Institut national de prévention et d'éducation pour la santé (INPES), 2011). Bien que les baluchonneuses soient des intervenantes, au regard de leur rôle et responsabilité, leur position s'apparente souvent à celle des aidants familiaux. L'intimité partagée jour et nuit avec la personne atteinte de DTA, la flexibilité et l'adaptation nécessaires à ce travail constituent des particularités qu'elles partagent

\footnotetext{
1Le terme présence-surveillance est utilisé pour la première fois dans la Politique de soutien à domicile Chez soi : le premier choix (MSSS, 2003) comme synonyme du terme « gardiennage » pour des adultes dépendants.
} 
avec les aidants familiaux et que ne vivent pas les autres intervenants. Or, cette expérience spécifique n'a pas encore été documentée. Ainsi, l'objectif de cet article est d'exposer la responsabilité endossée par les baluchonneuses offrant du répit aux aidants. La première partie aborde le contexte de l'étude qui visait à décrire le rôle des baluchonneuses dans la perspective d'un modèle théorique sur la responsabilité morale des aidants. La deuxième partie, la plus substantielle, se consacre à expliciter en quoi le rôle des baluchonneuses constitue une responsabilité morale. En conclusion, une brève réflexion sur la notion de responsabilité morale en contexte de soins est proposée.

\section{MODÈLE THÉORIQUE SUR LA RESPONSABILITÉ MORALE DES AIDANTS}

La notion de responsabilité est polysémique. En philosophie, on en parle en termes d'éthique de l'intersubjectivité au sens d'un souci des personnes sous sa responsabilité (Ricœur, 1994). Lévinas (2006), par exemple, postule que le lien qui unit un sujet à une personne vulnérable est essentiellement un rapport de responsabilité auquel on ne peut échapper à partir du moment où l'on rencontre le Visage de l'autre. Ces éthiques de la sollicitude « admettent que la possibilité de la vulnérabilité d'autrui nous prenne littéralement en otage ou que l'engagement envers autrui soit parfois inéluctable » (Métayer, 2001, p. 21). Selon Éthier (2012), la responsabilité des aidants familiaux en est une morale, empreinte de sollicitude, sans attente de réciprocité et au sein de laquelle subsistent néanmoins des aspects positifs et une liberté d'agir.

C'est sur ce postulat que s'est élaborée une étude qualitative visant à comprendre le processus de construction de la responsabilité morale des aidants à partir de la méthodologie de la théorisation enracinée. Au terme de l'étude, six composantes de la responsabilité morale des aidants familiaux ont été mises en lumière : 1) la nature des transformations de l'aidé dont celles touchant l'identité ; 2) la transformation de la relation aidant-aidé ; 3) les fondements sous-jacents à la prise de responsabilité dont l'amour, la promesse ou le devoir ; 4) l'actualisation de la responsabilité qui implique la résolution de dilemmes éthiques ; 5) l'évolution dans le temps de la réponse à l'appel de la responsabilité et 6) la singularisation de la responsabilité en dépit des pressions et des contraintes extérieures. Ces composantes sont interreliées et contribuent à faire du rôle d'aidant une responsabilité éminemment morale (Éthier, 2012 ; Éthier et al., 2014). Suite à cette étude, il semblait intéressant d'étudier si ces composantes étaient aussi présentes chez d'autres types d'aidants que familiaux. Bien que le travail des aidants et celui des baluchonneuses comportent des différences fondamentales, il était possible d'envisager que certaines composantes morales de leurs responsabilités soient partagées.

\section{MÉTHODE ET CONSIDÉRATIONS ÉTHIQUES}

Cette étude qualitative, exploratoire et descriptive, s'est réalisée à l'aide d'une collecte des données en trois temps dans un souci de triangulation des données. Dix baluchonneuses sélectionnées selon un échantillonnage non probabiliste de volontaires (Patton, 2002) ont participé à une entrevue individuelle semi-dirigée en face à face (temps 1) d'une durée de 60 à 90 minutes et à une entrevue téléphonique (temps 2) d'environ 30 minutes. Puis, elles se sont jointes à un groupe de discussion focalisée (temps 3) d'une durée de deux heures auquel dix autres baluchonneuses se sont ajoutées. Ainsi, les 20 baluchonneuses employées par BA au 
moment de l'étude y ont participé. Les questions des entrevues individuelles portaient sur les composantes de la responsabilité morale adaptées à leur réalité. Plus précisément, elles ont été interrogées sur: les fondements derrière leur choix de devenir baluchonneuses; la manière dont se déroule leur travail au quotidien ; leur évolution personnelle depuis leurs débuts dans ce travail ; la façon dont elles prennent des décisions dans des situations complexes ; et la relation qu'elles entretiennent avec l'aidé. Les questions d'entrevues téléphoniques portaient sur leur dernier baluchonnage (moins de deux jours après leur retour) et avaient pour objectif le partage de leur expérience à partir d'une situation concrète et récente. Ainsi, leur réflexion à l'égard de leur responsabilité morale avait pu cheminer entre les entrevues face à face et téléphonique amenant nuances et profondeur lors de cette seconde entrevue. Le groupe de discussion a permis de recueillir les réactions face aux résultats préliminaires qui leur ont été présentés. Les participantes au groupe de discussion ne savaient pas qui d'entre elles faisaient partie de l'échantillon ayant participé aux entrevues. Les baluchonneuses qui ne souhaitaient pas participer au groupe de discussion avaient la possibilité de prendre connaissance des résultats préliminaires et de quitter la salle par la suite. Une pause entre les deux activités leur en donnait l'occasion. Mais toutes sont restées et ont signé un formulaire de consentement à leur participation au groupe de discussion. Les entrevues individuelles et la discussion de groupe ont été enregistrées et retranscrites et ont fait l'objet d'une analyse de contenu en profondeur selon la perspective épistémologique mixte de Miles et Huberman Huberman et al. (2003). La grille d'analyse de base comprenait les cinq composantes de la responsabilité morale étudiées et abordées dans les entrevues, mais laissait place à l'émergence de sous-catégories et d'autres catégories provenant des données. Les entretiens téléphoniques ont été analysés à l'aide de la même grille lors d'écoutes attentives et systématiques des enregistrements audio. Cette étude a reçu l'approbation du Comité d'éthique de la recherche de l'Université Laval (CERUL) ( $N^{0}$ d'approbation 2013-027/12-03-2013) en mars 2013.

\section{RÉSULTATS}

\section{Description de l'échantillon}

L'âge moyen des vingt participantes était de 59 ans. Elles cumulaient entre une année et 11 années d'expérience de travail comme baluchonneuses, soit en moyenne quatre ans et neuf mois. Bien qu'elles provenaient de milieux professionnels différents (services, santé, arts), la majorité d'entre elles possédaient une expérience se rapprochant de plusieurs des tâches requises par l'emploi de baluchonneuses. Effectivement, elles ont été aidantes familiales, travailleuses sociales, infirmières ou préposées aux bénéficiaires avant de devenir baluchonneuses.

\section{Composantes de la responsabilité morale des baluchonneuses}

Les résultats de cette étude montrent que cinq des six composantes de la responsabilité morale des aidants d'Éthier (2012) s'appliquent également aux baluchonneuses. On y trouve les fondements derrière le choix de devenir baluchonneuses, l'actualisation de la responsabilité, l'évolution de la réponse à l'appel de la responsabilité, la singularisation de la responsabilité et la transformation de leur relation avec l'aidé. S'ajoutent cependant trois autres composantes. 
Premièrement, la variation de la relation entretenue avec les proches aidants. Ensuite, l'implication de la baluchonneuse dans la relation aidant-aidé. Troisièmement, la gestion de la médication et des soins fournis à l'aidé. Les huit composantes de la responsabilité morale, telles que vécues par les baluchonneuses, sont présentées dans la figure 1 et décrites dans les lignes qui suivent.

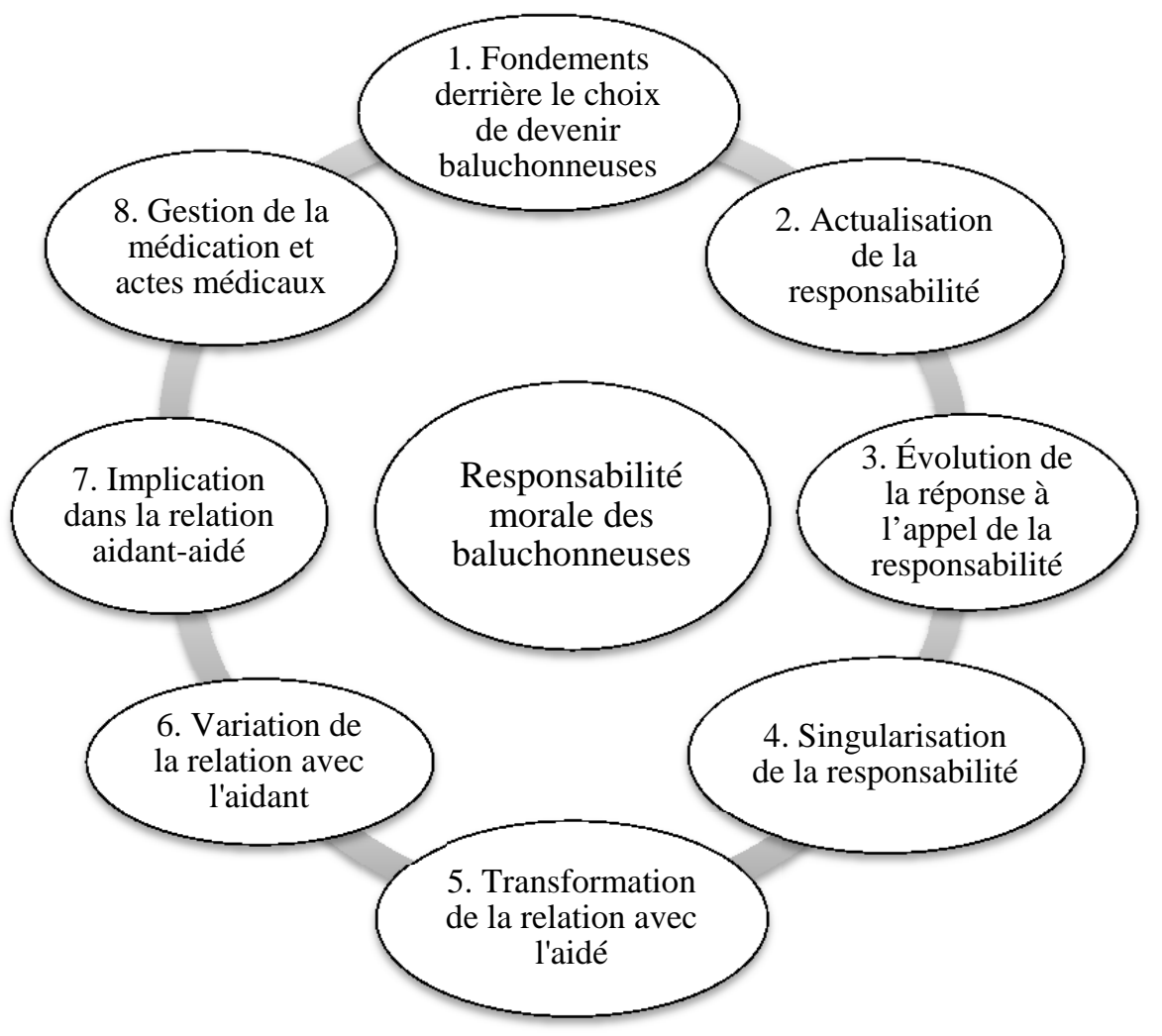

Figure 1 : Les composantes de la responsabilité morale des baluchonneuses

\section{Les fondements derrière le choix de devenir baluchonneuses}

Les fondements de la responsabilité réfèrent aux motivations profondes pour lesquelles les baluchonneuses ont décidé d'assumer ce rôle. Ces motivations sont de nature spirituelle, personnelle et matérielle. Au plan spirituel, ce sont principalement le sens du devoir, des motivations altruistes et une conception humaniste de l'existence qui ont incité les participantes à devenir baluchonneuses, "par solidarité de la grande chaîne humaine », disait l'une d'elles (Baluchonneuse 5). Certaines parlent même d'une forme de destin missionnaire : « C'est ma participation dans l'univers » (Baluchonneuse 8). «Baluchon, je te dirais que c'est la vie qui me l'a envoyé. Tu sais, dans la vie, on ne dirige pas tout. Moi, j'étais faite pour faire ça et je ne le savais pas » (Baluchonneuse 3). La dimension personnelle fait référence aux besoins individuels auxquels répond le baluchonnage : socialiser, se sentir utile et relever de nouveaux défis. La possibilité d'être active sur le marché du travail et de développer un lien d'appartenance à une organisation a aussi attiré des baluchonneuses. Ajoutons que la particularité de l'emploi, en termes d'horaires et de conditions de travail, est intéressante pour les personnes retraitées, celles qui ont un style de vie plutôt nomade ou celles qui apprécient 
gérer leur horaire. En ce qui a trait à la dimension matérielle, les baluchonneuses ne font pas exception : elles doivent gagner leur vie. Or, l'aspect monétaire, à lui seul, ne semble pas un motif suffisant pour occuper cet emploi. Disons que pour elles, le revenu de leur travail est essentiel, mais occupe une place de moindre importance dans l'échelle de leurs motivations.

Bien que soulevant aussi des motivations personnelles et matérielles, les motivations à devenir baluchonneuses sont prioritairement de nature spirituelle pour les participantes. Elles font preuve de sollicitude, d'empathie et d'un intérêt réel pour des êtres fragilisés au-delà de ce qu'il est généralement attendu d'un intervenant: «Là j'ai compris qu'il s'agissait d'un univers d'émotions puis d'intuitions. Alors j'ai dit, ça me convient, parce que c'est ça qui m'intéresse, moi, dans la vie, c'est de fonctionner avec le cœur »(Baluchonneuse 8). Ainsi, la nature des motivations orientant les baluchonneuses vers ce travail s'accorde avec la première composante de la responsabilité morale qui est la présence de fondements moraux.

\section{L'actualisation de la responsabilité}

La seconde composante de la responsabilité morale renvoie à la façon dont se réalise ce travail au quotidien. Le travail de baluchonneuse est caractérisé par des déplacements successifs et de multiples séjours dans des familles toujours différentes. Ainsi, l'actualisation de leur responsabilité est marquée par le changement perpétuel qui suppose une adaptation à plusieurs niveaux, tant relationnel, environnemental, temporel, qu'organisationnel et comporte des désagréments. Elles doivent en effet s'adapter à des familles vivant dans des environnements et des contextes nouveaux, s'ajuster à la durée du baluchonnage qui varie, ainsi que se conformer aux changements organisationnels chez BA. En plus, la nature de leur travail implique son lot de désagréments particuliers : "la première nuit, je ne dors pas beaucoup parce que, bon, la personne, elle se lève »(Baluchonneuse 2). Ce type de désagrément n'est pas vécu par les professionnels qui n'offrent que quelques heures de soins ou services par jour. Les baluchonneuses, de leur côté, font le choix de les accepter afin d'offrir un accompagnement cohérent avec leurs valeurs.

Justement, c'est la promotion de valeurs dans les gestes quotidiens qui donne un sens moral à leur responsabilité quotidienne. Certes, leur manière d'agir et de réagir reflète leur professionnalisme, mais illustre encore davantage les valeurs profondément humaines qui les animent. Pour elles, la priorité consiste à maintenir l'autonomie et à assurer la sécurité. Ensuite, il s'agira de porter un regard sensible sur la personne et ce qui la caractérise au-delà de la maladie. C'est en toutes circonstances qu'elles tenteront d'en préserver la dignité. Par contre, c'est dans la mesure du possible que d'autres valeurs, comme le maintien de l'intimité de l'aidé, seront respectées. En effet, l'actualisation de la responsabilité se manifeste par des dilemmes éthiques auxquels les participantes sont constamment confrontées qui consistent à établir la priorité dans un contexte où deux valeurs fondamentales s'opposent. Par exemple: « c'est important d'assurer sa sécurité, et en même temps, maintenir son autonomie si la personne est indépendante. II faut vraiment savoir juger de ça. II ne faut pas être collé à lui, il faut le surveiller, mais avec une certaine distance pour pas que la personne se sente assaillie » (Baluchonneuse 8). En somme, il est possible de constater que l'actualisation de la responsabilité de la baluchonneuse ne se limite pas à l'accomplissement de tâches de soins ou 
d'accompagnement dans un contexte particulier. Elle renvoie à une expérience morale où se côtoient valeurs et dilemmes éthiques.

\section{L'évolution de la réponse à l'appel à la responsabilité}

L'évolution de la réponse à l'appel de la responsabilité, troisième composante de la responsabilité morale, implique l'idée que la manière d'assumer leur responsabilité se transforme avec le temps, mais pas n'importe comment. Cette évolution comprend trois éléments: la transformation de soi, la (re)définition de limites à leur responsabilité et la recherche de changement. Les baluchonneuses reconnaissent que ce travail leur permet de découvrir leur force intérieure et constitue un terrain fertile pour une croissance personnelle inestimable: «Je me sens ressourcée, je me sens transformée, je me sens une nouvelle personne grâce à quelqu'un dont j'ai pris soin et qui m'a apporté beaucoup » (Baluchonneuse 4). Cette transformation de soi s'exprime également par une plus grande ouverture aux autres et un certain lâcher-prise pour se centrer sur ce qui compte le plus : la relation avec l'aidé. « Tu finis par comprendre que tout ce que tu avais prévu cette journée-là, à cette heure-là, ça ne se peut pas, ça ne sera pas sur l'agenda. On l'accueille. On prend ça d'où c'est rendu. Tu n'es pas en lutte avec ça » (Baluchonneuse 1). Les participantes rapportent l'importance pour elles de fixer des limites dans leurs baluchonnages. Ces limites ont évolué avec le temps et d'autres ont pu se rajouter. Dans l'ensemble, ces limites portent sur l'état de santé de l'aidé, sur la durée et le contexte de l'accompagnement ainsi que sur des limites plus personnelles. Ces dernières sont souvent en lien avec des expériences douloureuses vécues antérieurement auxquelles certains baluchonnages les confrontent, comme la présence de violence. De plus, des baluchonneuses mentionnent l'importance d'améliorer leur pratique et d'avoir de nouveaux défis à relever. La formation continue qu'offre BA semble satisfaire une partie de ces besoins. Plus encore, la nature changeante et diversifiée de leur travail ne leur cause pas que des désagréments: elle constitue un défi et leur permet d'évoluer de façon continue. Ces trois différentes réponses à l'appel de la responsabilité (transformation de soi, redéfinition de limites à leur responsabilité et besoin de changement) suggèrent que les baluchonneuses se laissent affecter de manière positive par leur emploi, sans toutefois se laisser envahir par leur engagement moral envers l'aidé.

\section{La singularisation de la responsabilité}

La quatrième composante est la singularisation de la responsabilité, laquelle fait ressortir la place centrale qu'occupent les baluchonneuses dans la prise de décision. À la différence de ce qu'il en est pour les aidants, le travail des baluchonneuses se situe dans un cadre professionnel. Par conséquent, il existe des conventions (règles ou normes) qui doivent être respectées au regard du rôle et des responsabilités qu'elles endossent. L'aidant fournit par ailleurs des renseignements sur la routine, les façons de faire et les goûts de l'aidé. Ces derniers peuvent également les guider. De plus, les baluchonneuses sont soutenues et encadrées par BA avec qui elles ont des contacts réguliers. Les personnes-ressources identifiées par les aidants, les divers professionnels, leurs collègues baluchonneuses, les pharmaciens, et les médecins constituent d'autres sources d'informations ponctuelles. Alors qu'elles tiennent généralement 
compte des attentes et des conseils provenant de l'extérieur, il arrive que leur expérience et leur intuition leur dictent d'autres façons de faire.

La famille me demandait de redonner une espèce de somnifère, ou un calmant, avec un autre verre de vin. Moi, je trouvais que ça ne marchait pas, que le vin avait plutôt tendance à exciter la personne, que ça avait un effet contradictoire. Je n'étais pas d'accord avec ça, du tout. Je craignais [...] l'effet négatif d'une surmédication. Et l'infirmière était d'accord avec moi. (Baluchonneuse 5)

C'est ainsi que les baluchonneuses parviennent à développer une manière singulière d'assumer leur responsabilité tout en tenant compte des contraintes externes et des conseils auxquels elles sont assujetties, car, ultimement, elles devront répondre de leurs actes. Cela constitue un élément de plus venant construire leur responsabilité morale à l'égard de l'aidé qu'elles accompagnent.

\section{La transformation de la relation avec l'aidé}

La cinquième composante de la responsabilité morale est la transformation de la relation entre l'aidé et la baluchonneuse. C'est notamment à travers cette relation que s'actualisent les notions de sollicitude et de non-réciprocité présentes dans la définition de la responsabilité morale de départ. Généralement, le rapport d'aide se caractérise par l'asymétrie qui vient du fait d'être responsable d'une personne vulnérable. Or, on constate qu'une certaine forme de réciprocité dans le rapport relationnel entretenu avec l'aidé s'installe au fil du temps. Les activités et les moments partagés contribuent au développement d'un fort lien de proximité, et ce, en dépit de l'asymétrie toujours présente. Ce lien se construit par l'échange de tendresse, de rire et d'intimité, créant ainsi une relation davantage réciproque. Cet exemple l'illustre parfaitement :

J'ai des moniteurs pour l'entendre si elle se réveille la nuit. Alors, je sais qu'elle ne dort pas. Je me suis dit : je vais aller coucher avec elle. Alors, je me couche avec elle. Un moment donné, je m'endors. Elle, tu la sens sécure quand tu es couchée à côté d'elle. Parce qu'elle avait tellement peur d'être toute seule dans sa maison! [...] Alors, un moment donné, elle n'était pas capable de dormir parce que je ronflais [rire] [...] On est tellement habituées ensemble qu'on n'a plus à s'excuser. On a pouffé de rire en pleine nuit! (Baluchonneuse 10)

La transformation dans la relation avec l'aidé atteste que la responsabilité envers une personne fragile n'est pas forcément et uniquement asymétrique. Bien qu'il n'y ait pas d'attentes de réciprocité au départ, le temps passé ensemble et l'intimité partagée tissent une relation nouvelle empreinte de réciprocité. En conséquence, les effets de la transformation de relation témoignent de l'enjeu moral de la responsabilité des baluchonneuses.

\section{La variation de la relation avec les aidants}

Une sixième composante s'est ajoutée aux résultats lors de l'analyse. Elle cible la relation entre les baluchonneuses et les aidants. En fait, cette relation s'est montrée cruciale et plus complexe qu'estimée initialement. La confiance mutuelle entre l'aidant et la baluchonneuse constitue un préalable à l'établissement de la relation au point où elle déterminera parfois si le baluchonnage aura lieu ou non. Bien qu'elles passent la majorité de leur temps avec les aidés, les 
baluchonneuses ont exprimé une grande préoccupation à l'égard des aidants : « C'est étonnant, parce que c'est cette personne-là [l'aidant] qu'on va aider le plus et c'est celle qu'on va connaître le moins » (Baluchonneuse 5). Cette relation qui, au départ, se veut une relation de travail, peut ainsi se transformer en relation d'amitié et se poursuivre au-delà du baluchonnage : « Ça devient comme une amie, ça devient comme la famille. Ce n'est plus "la baluchonneuse", c'est "une amie" » (Baluchonneuse 10). Des divergences, voire des tensions peuvent par ailleurs survenir. Des attentes différentes concernant l'accueil réservé à la baluchonneuse, le partage d'informations, les tâches et les responsabilités respectives peuvent en être la source :

Alors, maintenant, je leur dis aux aidants : ma responsabilité, c'est votre père ou votre mère, ou votre mari, ou votre femme, mais ce n'est pas le chien ou le chat. Je voudrais que vous sachiez que s'il part, le chat, le soir à six heures et puis qu'il faut que je fasse des choses avec monsieur, je ne vais pas passer trois heures à chercher le chat. (Baluchonneuse 4)

En somme, la relation entre aidants et baluchonneuses est soumise à un ensemble de facteurs de nature professionnelle et personnelle. Elle sera plus ou moins intime et agréable selon ce qui est perçu et attendu par chacun. Or, cette relation est fondamentale : c'est dans la mesure où la confiance s'installera dans la relation avec les baluchonneuses que les aidants partiront l'esprit tranquille. C'est donc la qualité de cette relation qui permettra le transfert de la responsabilité morale de l'aidant vers la baluchonneuse.

\section{L'implication dans la relation aidant-aidé}

Une septième composante fondamentale de la responsabilité morale ressort dans cette étude. Cette composante repose sur l'implication des baluchonneuses dans la relation aidant-aidé. Le lecteur sait maintenant que les baluchonneuses développent de façon individuelle des liens avec l'aidé ( $5^{\mathrm{e}}$ composante) et avec l'aidant ( $6^{\mathrm{e}}$ composante). Mais plus encore, les participantes soulignent l'importance de leur présence au sein de la dynamique établie entre l'aidant et son proche. De ce constat émerge la notion de rapport triadique. Par exemple, les baluchonneuses constatent la nature de la relation qu'entretiennent l'aidant et l'aidé : «Moi, je vais te dire quelque chose : l'aidant s'en va parce qu'il a besoin de répit. Mais pourrais-tu croire que l'aidé, il en a besoin, d'un répit, aussi ? Parce qu'ils développent une dynamique entre eux deux des fois » (Baluchonneuse 3). Qu'elle soit bonne ou mauvaise, cette relation aidant-aidé, même après le départ de l'aidant, continue d'influencer le travail des baluchonneuses. En outre, par le biais du journal de bord destiné aux aidants, les baluchonneuses consignent les informations pouvant alléger les tâches de l'aidant et faciliter le rapport avec son proche. Selon une baluchonneuse, « la dernière chose qu'on veut après un passage dans une famille, c'est qu'ils sentent qu'ils fassent mal les choses. Alors, c'est très délicat. Le journal qu'on fait après est très important, c'est une partie très délicate du travail » (Baluchonneuse 2).

De l'analyse, il ressort que la relation entre l'aidant et son proche influe sur la baluchonneuse et sa manière d'assumer sa responsabilité morale envers l'aidé. Pour cette raison, l'existence de cette triade doit être prise en compte pour une définition plus juste et une meilleure compréhension de la dimension morale de leur travail. 


\section{La gestion de la médication et des actes médicaux}

La huitième et dernière composante de la responsabilité morale, soit la gestion de la médication et des actes médicaux, correspond à une préoccupation exprimée par les baluchonneuses lors de la discussion de groupe. Elles disent se retrouver entre l'arbre et l'écorce. Comme les aidants, en partageant leur quotidien pendant plusieurs jours, elles sont des témoins privilégiés des impacts de la médication sur les personnes atteintes de DTA. Comme le font les aidants, les baluchonneuses peuvent être appelées à assumer d'importantes responsabilités relevant d'actes professionnels quant à l'administration de médicaments et de soins (injection d'insuline, ajustement de dosage d'un médicament, par exemple), et ce, sans toujours détenir la formation suffisante. Lorsque des observations les amènent à se questionner, par sécurité, elles préfèreront toutefois s'adresser à un professionnel de la santé. Bien que leur rôle dans ce domaine s'apparente à celui des aidants, reconnus pour prodiguer la grande majorité des soins requis par l'aidé, elles expriment un malaise devant les conséquences possibles pour elles en tant qu'intervenantes.

Lui [l'aidant], il peut le faire, il peut se tromper. Moi, je ne peux pas. Va falloir que je justifie. Alors même si on m'a dit : il [l'aidant] va préparer les dosettes, les capsules avant, je refuse. S'il se trompe, c'est moi qui l'a injecté ! Je ne veux pas de cette responsabilité-là que j'appelle morale. (Baluchonneuse du groupe de discussion)

Ainsi, la gestion de la médication et des soins médicaux, plus particulièrement des conséquences éventuelles d'une mauvaise gestion, relève pour les baluchonneuses d'une responsabilité morale qu'elles ont des réticences à endosser.

\section{DISCUSSION}

\section{Originalité de cette étude}

Le modèle unique de répit offert par $\mathrm{BA}$, en termes d'effets pour les aidants, a déjà été étudié (Gendron, 2001 ; Gendron et Adam, 2004 ; Lucet, 2011 ; Landry, 2013) et est cité en exemple dans un mémoire de la Chaire Desjardins en soins infirmiers à la personne âgée et à la famille dans le cadre des Consultations sur le Livre blanc pour la création d'une assurance autonomie (Ducharme et al., 2013). Or, le métier des baluchonneuses, quant à lui, n'avait pas encore été documenté. Cette étude aura permis de mettre en lumière ce travail singulier. Bien que les composantes de la responsabilité morale des aidants familiaux aient été récemment dégagées (Éthier, 2012 ; Éthier et al., 2014), la notion de responsabilité morale est également un champ de recherches et d'interventions peu exploré en service social. L'application des composantes de la responsabilité morale des aidants au travail des baluchonneuses donne une couleur particulière à cette étude. Cette approche aura permis de dépasser la simple description des tâches des baluchonneuses pour dévoiler la présence effective d'une responsabilité morale déterminée par huit composantes, dont trois leur étant spécifiques. Les résultats montrent que les baluchonneuses endossent elles aussi une responsabilité morale. En conséquence, elles connaissent mieux que quiconque l'ampleur de celle qui incombe aux aidants familiaux. Ainsi, il ne sera plus possible de parler du baluchonnage comme d'un simple travail d'accompagnement dans un objectif de répit de l'aidant. Prendre soin d'un proche atteint de DTA est une 
responsabilité morale que les aidants confient aux baluchonneuses et qu'elles assument entièrement comme telle.

\section{Limites de cette étude}

Les principales limites à cette étude sont en lien avec la collecte de données. Quelques entrevues en face à face se sont tenues dans des lieux publics (restaurant, café, centre commercial) dont le bruit ambiant présent a pu nuire à la concentration des participantes. Aussi, compte tenu du territoire à couvrir (l'ensemble du Québec), deux auxiliaires de recherche ont mené les entrevues. Bien que le canevas d'entrevue fût le même, comme il s'agissait d'entrevues semi-structurées, il est possible que des différences dans la façon de mener les entrevues aient induit des variations dans les réponses.

\section{Apport de cette étude à la réflexion sur la responsabilité morale et la pratique auprès des aidants}

La présente étude apporte au moins trois éléments nouveaux à la réflexion sur la responsabilité morale: les baluchonneuses assument une responsabilité morale; l'engagement dans le traitement pharmacologique en fait partie ; et les interventions futures devraient se consacrer à diminuer le fardeau moral des aidants. Premièrement, il est reconnu que la responsabilité envers une personne atteinte de DTA renvoie à une expérience morale où se côtoient solidarité, responsabilités, obligations et dilemmes éthiques (Villeneuve, 2008 ; Lin et al., 2012). Ce que confirme cette étude. Contrairement à ce que l'on pourrait croire, le sentiment de responsabilité envers l'autre n'aliène pas l'individu, mais éveille plutôt en lui des possibilités humaines des plus irremplaçables et le transporte dans l'infini d'une relation privilégiée qu'il ne pourra jamais épuiser (Habib, 1998). La relation avec l'aidé est effectivement un enjeu fondamental du travail de soins mentionné dans plusieurs études (Armstrong-Stassen et Cameron, 2005 ; Gagnon, 2005 ; Gagnon, 2006 ; Pitrou et al., 2006 ; Grosclaude, 2007 ; INPES, 2011). Le degré d'intimité partagée avec l'aidé influera entre autres sur la manière dont sera assumée la responsabilité envers lui (Davies et al., 2010 ; Harris et al., 2011). On parlera de continuité ou de réciprocité relationnelle, ou, au contraire, de détachement ou de sentiment d'obligation de prendre soin (Ablitt et al., 2009). Holstein (2001) souligne que, dans ce sens, l'aidant partage un univers moral avec son proche, composé entre autres de rapports affectifs. II insiste sur l'impératif de saisir cet univers pour comprendre le travail moral requis par l'aidant. Ainsi, cette étude met en lumière l'univers moral des baluchonneuses et avance en conséquence qu'elles assument une responsabilité morale. Deuxièmement, lors de la discussion de groupe, les baluchonneuses ont abordé la responsabilité morale en regard de la gestion de la médication. Elles ont ainsi fait ressortir que l'engagement dans le traitement pharmacologique n'est pas un processus sousjacent, ou consécutif à la responsabilité morale tel qu'envisagé dans une étude antérieure (Éthier, 2012). Cet engagement en constitue plutôt une des composantes au même titre que les autres. Effectivement, gérer la médication et les soins médicaux implique en soi une grande responsabilité morale. À titre d'exemple, comme l'ont souligné les baluchonneuses, choisir ou non de donner un médicament et déterminer le meilleur moment pour le faire, c'est prendre un certain risque dont les implications peuvent être considérables. Les résultats de cette étude confirment donc le besoin de sensibiliser les professionnels de la santé aux enjeux impliqués 
dans la gestion du traitement pharmacologique d'une tierce personne, dont la logistique, l'administration du médicament et la sécurité (Travis et al., 2000), afin que ces professionnels puissent accompagner et soutenir les aidants, familiaux ou non, dans cette responsabilité. Troisièmement, grâce à cette étude, il est permis de croire que la responsabilité morale de l'aidant familial puisse se partager, du moins en partie. Conséquemment, il est envisageable de chercher des manières d'alléger le fardeau de cette responsabilité morale. L'agir responsable des aidants familiaux, tout comme celui des baluchonneuses, prend une dimension hautement morale mariant à la fois une certaine obligation de faire à une obligation d'être qui est présente et pourtant peu documentée. D'autres études devront donc être entreprises afin de mettre l'accent sur les impacts concrets d'une telle responsabilité morale et aussi pour permettre aux aidants de saisir la nature réelle de leur engagement pour ne pas s'y laisser engloutir. Reconnaître qu'il s'agit d'une responsabilité morale est le premier pas vers la diminution de ses conséquences. Le second consiste à développer des stratégies pour y faire face, pour consentir à en déléguer une partie et parfois même pour se donner le droit de la refuser.

ÉTHIER, Sophie Professeure agrégée École de service social Université Laval

GAGNON-GRÉGOIRE, Catherine Auxiliaire de recherche École de service social Université Laval

DUPONT, Janine Détentrice d'une maitrise en gérontologie Université de Sherbrooke

\section{BIBLIOGRAPHIE}

Ablitt, A., G.V. Jones et J. Muers (2009). « Living with Dementia: A Systematic Review of the Influence of Relationship Factors », Aging and Mental Health, vol. 13, n² 4, p. 497-511.

Armstrong-Stassen, M., et S. Cameron (2005). «Concerns, Satisfaction, and Retention of Canadian Community Health Nurses », Journal of Community Health Nursing, vol. 22, $\mathrm{n}^{\circ} 4$, p. 181-194.

ASSSCN (Agence de la santé et des services sociaux de la Capitale-Nationale) (2011). Programme hébergement temporaire - Région de la Capitale-Nationale, Cadre de référence, ASSS de la Capitale-Nationale, Direction régionale des programmes clientèles.

Bégin, A., D. Bouchard et D. Goulet (2004). Soutenir les proches aidants. Constats, enseignements, pistes de réflexion: vingt-sept expériences de répit, de formation et de soutien, Fonds de partenariat sur la maladie d'Alzheimer et les affections connexes [http://publications.msss.gouv.qc.ca/acrobat/f/documentation/2004/rapfonds.pdf] 
Binhas, L. (2010). Les besoins des proches aidants, Table régionale de concertation des ainéEs du Centre-du-Québec.

Caisse nationale de solidarité pour l'autonomie (CNSA) (2008). Synthèse du rapport d'étude sur l'analyse des besoins des aidants familiaux. Du diagnostic des déséquilibres à l'expression des besoins, Paris, CNSA.

Damasse, J., É. Gagnon et A. Larochelle (2003). Une source alternative de répit. Rapport d'évaluation du service « Répit-accompagnement », CLSC-CHSLD Haute-Ville-Des-Rivières [http://www.csssvc.qc.ca/telechargement.php?id=52]

Davies, H.D., L.A. Newkirk, C.B. Pitts, C.A. Coughlin, S.B. Sridhar, L.M. Zeiss et A.M. Zeiss (2010). "The Impact of Dementia and Mild Memory Impairment (MMI) on Intimacy and Sexuality in Spousal Relationships », International Psychogeriatrics, vol. 22, nº 4, p. 618-628.

Ducharme, F., L. Lévesque, A. Bourbonnais, V. Dubé, et D. Saulnier (2013). Soutenir les proches aidants de personnes en perte d'autonomie... des avenues à considérer, Mémoire présenté au ministre de la Santé et des Services sociaux et ministre responsable des Aînés portant sur la création d'une assurance autonomie par la Chaire Desjardins en soins infirmiers à la personne âgée et à la famille.

Éthier, S. (2012). L'engagement des aidants dans le traitement pharmacologique de la maladie d'Alzheimer. Une expérience construite sur la responsabilité morale à l'égard de leur proche, thèse de doctorat, Sherbrooke, Université de Sherbrooke.

Éthier, S., et M.-R. Corbeil (2003). Évaluation du projet-pilote de répit-gardiennage au bénéfice d'aidants naturels cohabitant avec des personnes âgées en perte d'autonomie. Rapport final. Régie régionale de la santé et des services sociaux de Laval.

Éthier, S., A.-M. Boire-Lavigne et S. Garon (2014). «Plus qu'un rôle d'aidant. S'engager à prendre soin d'un proche atteint de la maladie d'Alzheimer est une responsabilité morale », Vie et Vieillissement, vol. 11, $\mathrm{n}^{\circ} 3$, p. 5-13.

Gagnon, É. (2005). «Autonomie ou bienfaisance ? Des obligations à la responsabilité », dans F.-R. Ouellette, R. Joyal et R. Hurtubise (dir.) Familles en mouvance. Quels enjeux éthiques?, Sainte-Foy, Presses de l'Université Laval, coll. « Culture et Société », p. 349-355.

Gagnon, É. (2006). « La responsabilité comme promesse », dans É. Gagnon et F. Saillant (dir.), De la responsabilité. Éthique et politique, Montréal, Liber, p. 57-67.

Gendron, M. (2001). «Baluchon Alzheimer. Un service de répit et d'accompagnement à domicile offert aux aidants des familles dont un des proches est atteint de la maladie d'Alzheimer », Revue canadienne de la maladie d'Alzheimer, vol. 4, n 4, p.4-7.

Gendron, M., et Adam, E. (2004). «Baluchon Alzheimer. An innovative respite service in the home of the natural caregiver of a family member suffering from Alzheimer's Disease », Alzheimer's Care Quarterly, vol. 6, n³, p. 249-261.

Grosclaude, M. (2007). «Soignants en gériatrie et maladie d'Alzheimer. Savoirs, représentations et usages, à partir d'une enquête », Psychologie et neuropsychiatrie du vieillissement, vol. $5, \mathrm{n}^{\circ} 2$, p. 139-152. 
Habib, S. (1998). La responsabilité chez Sartre et Lévinas, Paris, L'Harmattan.

Harris, S.M., M.S. Adams, M. Zubatsky et M. White (2011). "A Caregiver Perspective of How Alzheimer's Disease and Related Disorders Affect Couple Intimacy », Aging \& Mental Health, vol. 15, $\mathrm{n}^{\circ} 8$, p. 950-960.

Holstein, M. (2001). «To Inhabit a Livable Moral World: Mrs Dodge and her Caregivers », Alzheimer's Care Quarterly, p. 56-63.

INPES (Institut national de prévention et d'éducation pour la santé) (2011). Les pratiques et perceptions des aidants professionnels dans la prise en charge des patients atteints de la maladie d'Alzheimer. Synthèse de l'étude qualitative sur les aidants professionnels (EQAP), [http://alzheimer.inpes.fr/pdf/fr/synthese-aidant-quali.pdf].

Jansen, L., D.A. Forbes, M. Markle-Reid, P. Hawranik, D. Kingston, S. Peacock, S. Henderson, et B. Leipert (2009). « Formal Care Providers Perceptions of Home-and Community-Based Services: Informing Dementia Care Quality », Home Health Care Services Quarterly, vol. 28, $\mathrm{n}^{0} 1$, p. 1-23.

Landry, M. (2013). Évaluation d'un service de répit et d'accompagnement à domicile offert aux proches aidants de personnes atteintes de la maladie d'Alzheimer. Baluchon Alzheimer, thèse de doctorat, Sherbrooke, Université de Sherbrooke.

Lévinas, E. (2006). Totalité et infini. Essai sur l'extériorité, Paris, Librairie générale française.

Lin, M.-C., M. Macmillan et N. Brown (2012). « A Grounded Theory Longitudinal Study of Carers' Experiences of Caring for People with Dementia », Dementia, vol. 11, $\mathrm{n}^{\circ} 2$, p. 181-197.

Lucet, F. (2011). Maladie d'Alzheimer et Troubles Apparentés, Approches cognitives, psychothérapeutiques, comportementales, environnementales et éducatives. Le modèle du Baluchon Alzheimer est-il adaptable en France ?, mémoire. Université de Paris,

[http://www.lamaisondelautonomie.com/wpcontent/files/Le Baluchon Alzheimer est il adaptable en France Mmoire F Lucet 2012 FINAL.pdf]

Mackenzie, C.S., et G. Peragine (2003). «Measuring and enhancing self-efficacy among professional caregivers of individuals with dementia », American Journal of Alzheimer's Disease and Other Dementias, vol. 18, nº 5, p. 291-299.

Métayer, M. (2001). «Vers une pragmatique de la responsabilité morale », Lien social et politiques, vol. 46, p. 19-30.

Miles, M.B. et Huberman, M. (2003). Analyse des données qualitatives, $2^{\mathrm{e}}$ éd., Bruxelles, De Boeck.

MSCS (Ministère des Solidarités et de la Cohésion sociale) (2011). Formules innovantes de répit et de soutien des aidants. Guide pratique à destination des porteurs de projets,

[http://www.plan-alzheimer.gouv.fr/IMG/pdf/Guide_pratique_alzheimer_innovant.pdf].

MSSS (Ministère de la Santé et des Services sociaux) (2003). Chez soi : le premier choix. La politique de soutien à domicile, Gouvernement du Québec. 
MSSS (Ministère de la Santé et des Services sociaux) (2012). Vieillir et vivre ensemble. Chez soi, dans sa communauté, au Québec, Gouvernement du Québec.

Patton, M.Q. (2002). Qualitative Research \& Evaluation Methods, Thousand Oaks, Sage Publication.

Pitrou, I., M. Drouet, J. Ladner, Y. Moynot et P. Czernichow (2006). « Maladie d'Alzheimer, profil et besoins des aidants professionnels », Soins Gérontologie, n 59, p. 19-24.

Racine, M.-M. (2012). «Vers une actualisation des services de centre de jour pour les personnes âgées », Transphère, vol. 8, $n^{\circ} 1$, p. 36-38.

Ricœur, P. (1994). « Le concept de responsabilité. Essai d'analyse sémantique », Esprit, p. 2848.

Savard, J. (2008). L'utilisation des services de centre de jour par les personnes âgées qui présentent des incapacités, Thèse (Ph.D.), Université de Montréal.

Travis, S.S., L.S. Bethea et P. Winn (2000). « Medication Administration Hassles Reported by Family Caregivers of Dependent Elderly Persons », Journal of Gerontology, vol. 55, série A «Biological Sciences and Medical Sciences », $n^{\circ}$ 7, p. M412-M417.

Villeneuve, J. (2008). Pour un équilibre vital : des responsabilités équitables. Avis sur l'état de la situation des proches aidants auprès des personnes âgées en perte d'autonomie. Québec, Gouvernement du Québec, Conseil des aînés. 